Universitario de Valladolid with number (CEIm PI 19/1438). All patients signed the inform consent to enter the study.

Results Statistically significant differences were found in the rate of infection with respect to sex; being more frequent the infections in the feminine sex $(p=0.042)$. Marginally significant differences were found between the taking of NSAIDs and infection of the prosthesis $(p=0.1)$ although there was no statistical significance between the taking of NSAIDs and the analytical parameters of infection. This study demonstrates that periarticular infiltration does not increase the risk of infection $(p<0.05)$

Conclusions The use of NSAIDs has been associated with an immunosuppressive effect that leads to an increased risk of infection in total knee arthroplasty, so its use in the preoperative period should be limited. Female gender is other significative risk factor too. Our retrospective study has shown relationship between AINEs use and infection after knee arthroplasty.No increased risk of bleeding with AINEs in this sample.

\section{CONTINUOUS ERECTOR SPINAE PLANE BLOCK FOR POSTOPERATIVE ANALGESIA AS AN ALTERNATIVE TO EPIDURAL CATHETER IN THORACIC SURGERY FOR PATIENTS WITH EMPYEMA: A CASE SERIES}

C Simon*, N Parisi, G Samouri. Clinique Saint-Pierre, Ottignies, Belgium

\subsection{6/rapm-2021-ESRA.232}

Background and Aims We present a case series of five patients undergoing video-assisted thoracoscopic surgery for empyema. In this infectious state, we chose to perform a continuous ESP because of the relative contraindication of epidural catheter. We performed it to prevent and relieve the thoracic pain but also to limit the opioids consumption and their side effects in our frailty patients.

Methods The plan was general anaesthesia combined with a continuous unilateral ESP block. Postoperative analgesia was achieved by continuous infusion + bolus of L-Bupivacaine $0.125 \%$. Complementary analgesia was done with paracetamol and when possible NSAID. Patients received iv doses of piritramide in the recovery room, but no patient-controlled analgesia or other intravenous opioids was administered subsequently.

Results Most of the patients maintained a Numeric pain Rating Scale (NRS) between 0 and 3.

We removed the catheters the same day than the pleural drains, usually at day 4 . In the postoperative period, no side

\section{DAILY EVOLUTION OF NRS}

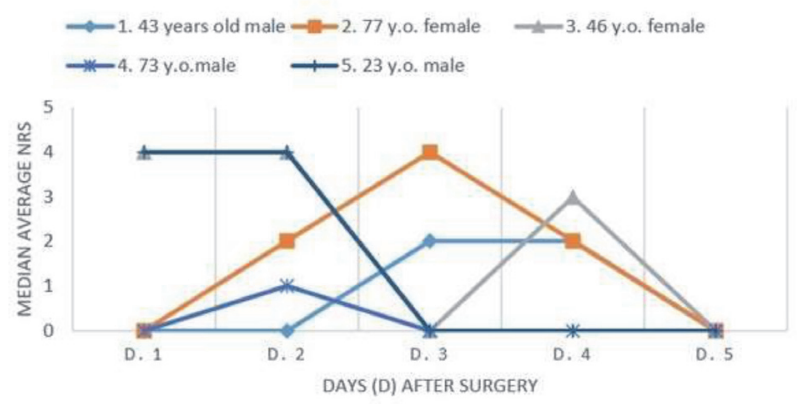

effects (motor blockade, urinary retention or hypotension) were noticed and patients attested high satisfaction scores. We emphasize the fact that none of them requested additional opioids.

Conclusions In cases of empyema, the ESP catheter combined with multimodal analgesia seems to be a safe and effective alternative to epidural analgesia for VATS surgery. This technique, easy to perform, allows an early mobilization of the patient regarding the low pain scores and the absence of side effects. Prospective randomized trials are needed to confirm the benefits of the ESPB compared to epidural or paravertebral bloc.

\section{PECS II BLOCK FOR ONCOLOGIC BREAST SURGERY DURING PREGNANCY: A PATIENT-CENTERED CASE REPORT}

1J Pinho*, ${ }^{2} \mathrm{~J}$ Moita, ${ }^{1} \mathrm{~N}$ Veiga. ${ }^{1}$ Francisco Gentil Portuguese Oncology Institute of Lisbon, Department of Anesthesiology, Lisbon, Portugal; '2Setúbal Hospital Center, Department of Anesthesiology, Setúbal, Portugal

\subsection{6/rapm-2021-ESRA.233}

Background and Aims Anesthesia and analgesia for surgery during pregnancy has focused on avoiding teratogenic drugs, preserving intrauterine homeostasis, and decreasing the risk of preterm labor. Data on the use of regional techniques and on patient preferences are still scarce. Based on a clinical case, we aimed to explore the patient experience of analgesia for oncologic breast surgery during pregnancy.

Methods A 19-weeks-pregnant 40-year-old woman with a diagnosis of breast cancer presented for mastectomy and sentinel lymph node biopsy. After discussion with the patient, an analgesia plan of post-induction pectoral type-2 block, acetaminophen, low-dose morphine, and postoperative acetaminophen, tramadol and prn metamizole was chosen. Acetaminophen and prn metamizole were prescribed home. Patient experience was elicited during the perioperative care and in a semi-structured interview at four-months.

Results On the first postoperative day, the patient reported only mild pain on movement and declined analgesia except for one administration of acetaminophen. After discharge, she reported significant pain on the surgical drain site. Until drain removal on the fourth postoperative day, she took acetaminophen and one prn metamizole. The main themes identified in the patient experience were a prioritization of fetal wellbeing, a concern for the effect of analgesic drugs on the fetus, and apprehension regarding nausea and sedation.

Conclusions Outcomes commonly measured in medical research are ultimately surrogates for outcomes that are more relevant to patients. This case suggests that a decreased need

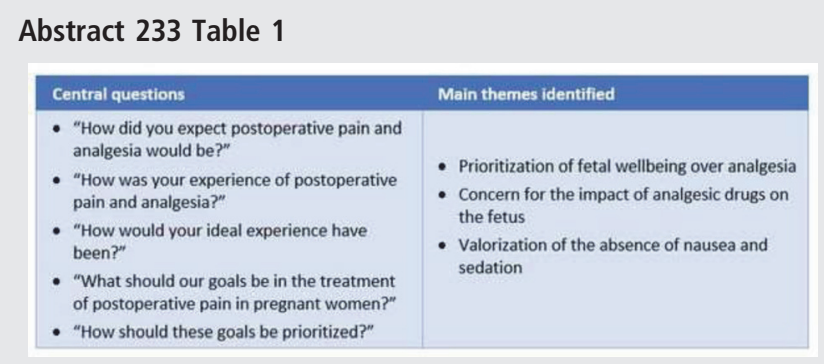


for analgesics may be more important to some patients than the absence of pain. Selected single-shot regional techniques are potentially useful in attaining this outcome.

\section{CONTINUOUS ERECTOR SPINAE PLANE BLOCK FOR PAIN MANAGEMENT IN OPEN RESECTION OF LOCALLY RECURRENT RENAL CELL CARCINOMA - A CASE- REPORT}

${ }^{1}$ AP Pereira* ${ }^{2}{ }^{2}$ Lobo, ${ }^{1} \mathrm{~F}$ Teixeira, ${ }^{1} \mathrm{R}$ Abrunhosa. ${ }^{1}$ Centro Hospitalar de Trás-os-Montes e Alto Douro, Vila Real, Portugal; ${ }^{2}$ Hospital das Forças Armadas - Pólo Porto, Porto, Portugal

\subsection{6/rapm-2021-ESRA.234}

Background and Aims Main issues in postoperative pain management for patients with single kidney include drug dose adjustment and further damage avoidance. Originally described for thoracic analgesia ${ }^{1}$, the erector spinae plane block (ESPB) can provide abdominal somatic and visceral analgesia ${ }^{2,3}$ and circumvent limitations in these patients. We describe the successful use of a continuous ESPB to provide effective perioperative analgesia for renal fossa surgery.

Methods A 79-year-old woman, ASA III, who underwent right nephrectomy due to renal cell carcinoma 4 years before, presented to the operating room for an open resection of renalbed carcinoma recurrence (subhepatic and abdominal wall).

Following general anaesthesia induction, an ultrasoundguided ipsilateral ESPB at T9 level (with $20 \mathrm{ml}$ ropivacaine $0,5 \%$ ) was performed and a catheter was inserted under ultrasound vision. During surgery IV dexamethasone $4 \mathrm{mg}$, fentanyl $0,05 \mathrm{mg}$, paracetamol $1 \mathrm{~g}$ and tramadol $50 \mathrm{mg}$ were administered.

For postoperative pain management we elected IV paracetamol and a ropivacaine $0,2 \%$ ESPB patient-controlled-analgesia device.

Results Patient underwent surgery uneventfully (mean arterial pressure $68-75 \mathrm{mmHg}$ and heart rate $55-68 / \mathrm{min}$ ). She was comfortable after the procedure and safely discharged from anesthesia care. The catheter was used for 2 days and pain was described as 'none' (at rest) or 'mild' (at movement) during vigilance. No rescue opioid was administered. Patient was satisfied with the approach and no postoperative complications were observed.
Conclusions The ESPB appears to be a useful alternative to epidural, paravertebral block or IV strategies for pain management in moderately aggressive retroperitoneal surgeries, with optimal safety profile and extent of sensitive coverage.

\section{EIGHT MG OF EPIDURAL MORPHINE: HOW MUCH SHOULD WE WORRY?}

R Rodrigues Oliveira, A Vasconcelos Pereira*, R Mendes de Carvalho, M Azevedo Coutinho. Hospital Vila Franca de Xira, Vila Franca de Xira, Portugal

\subsection{6/rapm-2021-ESRA.235}

Background and Aims Currently, administering a bolus of 2 to $3 \mathrm{mg}$ of epidural morphine for cesarean section postoperative pain management is widely recommended. Assuming that by using higher doses the increased side effects incidence will outweight any possible benefits in analgesic outcome, there is actually a lack of literature reporting higher dose regimens. We present a case of a patient who accidentally received a dose of $8 \mathrm{mg}$.

Methods A twenty-six-year-old, ASA II female underwent a cesarean section under epidural block due to fetopelvic disproportion. The epidural catheter was previously placed and used for labor analgesia. At the end of the surgery, a bolus of 8 mg of epidural morphine was mistakenly administered instead of $2 \mathrm{mg}$.

The patient was kept in the postoperative care unit for 18 hours and subsequently transferred to the ward. Ondansetron $4 \mathrm{mg} 8 / 8 \mathrm{~h}$ was prescribed for nausea and vomiting prophylaxis.

Results During the $24 \mathrm{~h}$ after the administration, adverse effects such as sedation, nausea, vomiting, pruritus, or urinary retention did not occur. She had no ventilatory depression and was hemodynamically stable throughout the day. Analgesia was optimal without the need of rescue analgesics.

Conclusions Despite the need of adequate monitoring, administering an higher dose of epidural morphine provided optimal analgesia without any adverse effects and allowed for an otherwise normal postoperative period. More studies might be needed in order to determine the actual safety of higher dose epidural morphine and its possible benefits for selected cases. 